\title{
THE GRANULE SIZE DISTRIBUTION INFLUENCE IN NANOCOMPOSITES ON OPTICAL AND MAGNETOOPTICAL SPECTRA
}

\author{
Alexey Yurasov ${ }^{1 *}$, Elena Gan'shina ${ }^{2}$, Alexey Sokolov ${ }^{1}$, Nikita Granovsky ${ }^{1}$ and Daria Zazymkina ${ }^{1}$ \\ ${ }^{1}$ Moscow Technological University (MIREA), Physical technological institute, 119454, Moscow, Russia \\ ${ }^{2}$ Moscow State University by M.V. Lomonosov (MSU), Physical Department, 119991, Moscow, Russia
}

\begin{abstract}
We have investigated the size effect (quasi-classical size effect) in nanocomposites. It was shown that the size effect can change the amplitude, form and sign of the optical and magnetooptical spectra. We have deduced formulas for size effect and discussed the applications of the distributions for corrected description of optical and magnetooptical properties with regard to the granule size effect. It is very important to consider the distribution on the granule size in size effect. This fact allows to describe optical and magnetooptical spectra of nanocomposites better, especially in near IR due to intraband electron transitions. We have deduced formulas for size effect and discussed applications of the distributions for corrected description of optical and magnetooptical properties with regard to the effect of the granule size.
\end{abstract}

\section{Introduction}

Optical and magnetooptical features in nanocomposites are strictly connected with the size effects [1-3]. So as there is a very important problem to describe optical and magnetooptical spectra of these structures especially in near IR due to intraband electron transitions. It was shown that the size effect can change the amplitude, form and sign of the optical and magnetooptical spectra[2,4]. The calculations with consideration of the distribution on the granule size in size effect allow to describe magnetooptical spectra for layerwise sprayed $\left(\mathrm{Co}_{40} \mathrm{Fe}_{40} \mathrm{~B}_{20}\right)_{\mathrm{x}}\left(\mathrm{SiO}_{2}\right)_{100-\mathrm{x}}$ nanocomposites qualitatively. Nanocomposites are heterogeneous magnetic materials in which ferromagnetic particles are placed in a para - or diamagnetic matrix of the dielectric. Nanocomposites are interesting because of the presence of a percolation threshold near which the electrical, optical and magneto-optical properties change significantly. This structure is a good example for the consideration of this phenomenon[1,2].

To describe the properties of ferromagnetic nanocomposites effective medium approaches[1] are used. It is necessary to consider that the scattering on the surface of the granules, leading to quasi-classical size effect, modifies as a diagonal $\varepsilon_{x x}=\varepsilon=\varepsilon_{1}-i \cdot \varepsilon_{2}$, and the nondiagonal $\varepsilon_{x y}=\gamma$ components of the tensor of the dielectric granule permittivity, if they are of average size (radius $r_{0}$ ) comparable with the free path length of electron $l$. The size effect has a significant influence on the optical and magneto-optical properties of nanocomposites particularly in the near infrared region of the spectrum by changing the amplitude, shape and the sign of the spectra. The present work discusses the influence of granule size distribution on the optical and magneto-optical properties of nanocomposites.

\section{Experimental data}

Nanocomposite "amorphous ferromagnet - insulator" $\left(\mathrm{Co}_{41} \mathrm{Fe}_{39} \mathrm{~B}_{20}\right)_{x}\left(\mathrm{SiO}_{2}\right)_{100-\mathrm{x}}$ was obtained in Voronezh state technical University by the method of ion-beam sputtering in argon atmosphere. The choice of such a complex granule composition $\mathrm{Co}_{41} \mathrm{Fe}_{39} \mathrm{~B}_{20}$ was made due to the fact that the amorphous structure of such a ferromagnetic is stable at room temperature. The specimen is produced by ion-beam sputtering in argon atmosphere. When using a vacuum chamber with three sources ion-beam [5]. 
A composite alloy target was used for the deposition. Floatable target $\mathrm{Co}_{41} \mathrm{Fe}_{39} \mathrm{~B}_{20}$ is manufactured by an induction vacuum melting. Cobalt with the purity $99.98 \%$ is used for the preparation of alloys, the amount of the carbonyl iron and boron correspond to the alloy composition. The composite target was an alloy target attached to the surface plate of a single crystal quartz/alumina. The thickness of the obtained film samples was $0.15-6.5 \mu \mathrm{m}$.

The composition of the resulting composites was controlled by the electron probe x-ray microanalysis. The electrical and magnetoresistive properties of the obtained samples were studied by out two-probe potentiometric method.

The measurement of TKE was carried out by the dynamic method, consisting in the detection of the small changes in the intensity of the reflected light when the sample is remagnetized by an alternating magnetic field [see, e.g. 6]. All other experimental details are shown in [6].

\section{Calculations and discussion}

We'll find a given size granule distribution in the following way[1]. We considered the granules as sphere particles with size $r_{0}$. The free time path of electrons in the granule $\left(\tau_{\text {part }}\right)$ is less than its corresponding time in the massive sample $\left(\tau_{\text {bulk }}\right)$ due to the collisions with the surface of the granules [1]:

$$
\frac{1}{\tau_{\text {part }}}=\frac{1}{\tau_{\text {bulk }}}+\frac{v_{f}}{r_{0}},
$$

where $v_{f}$ is Fermi velocity and

$$
\varepsilon_{\text {mod }}=\varepsilon+\frac{\omega_{p}^{2}}{\omega\left(\omega+i / \tau_{\text {bulk }}\right)}-\frac{\omega_{p}^{2}}{\omega\left(\omega+i / \tau_{\text {part }}\right)}
$$

The mentioned above values are the diagonal components of the permittivity tensor with the size effect influence $\varepsilon_{\text {mod }}$, where $\omega$ is frequency of the light, $\omega_{p}$ is plasma frequency.

Then, taking into account that the frequency dependence of the intraband conductivity can be described by the Drude-Lorentz formulas, similar to the formula [1] is the following:

$$
\begin{aligned}
& \gamma_{\bmod }=\gamma+ \\
& \frac{4 \pi \sigma_{x y}{ }^{b u l k}(0) / \tau_{b u l k}^{2}}{\omega\left(\omega+i / \tau_{\text {bulk }}\right)^{2}}-\frac{4 \pi \sigma_{x y} g r_{(0) / \tau_{p a r t}^{2}}}{\omega\left(\omega+i / \tau_{\text {part }}\right)^{2}}
\end{aligned}
$$

They are nondiagonal components of the permittivity tensor with the size effect influence,

$$
\begin{aligned}
& \text { where } \quad \sigma_{x y}^{\text {bulk }}(0)=4 \pi M_{s} R_{b u l k} / \rho_{b u l k}^{2}, \\
& \sigma_{x y}^{g r}(0)=4 \pi M_{s} R_{g r} / \rho_{g r}^{2}, M_{\mathrm{s}} \text { is }
\end{aligned}
$$
saturation magnetization, $R_{g r}$ is the coefficient of anomalous Hall effect, $\tau_{b u l k}$ is the corresponding free time of the electrons in the bulk material, $\tau_{g r}$ is the corresponding free time of the electrons in the granule, $\rho_{\text {bulk }}$ is the resistance of the bulk material, $\rho_{g r}$ is the resistance of the granules[4].

The size effect has an impact on the coefficient of the extraordinary Hall effect, and resistivity. The latter is given by the expression $\rho_{g r}=\rho_{\text {bulk }}\left(1+l / r_{0}\right)$, and the influence of the size effect on factor of the extraordinary Hall effect granules can be written as:

$$
R_{g r}=R_{\text {bulk }}+0.2 R_{s} \frac{l}{r_{0}}\left(1+\frac{l}{r_{0}}\right) \text {, }
$$

where $R_{s}$ is the value of the coefficient of the extraordinary Hall effect on the surface material of the granules [7]. The characteristic dependences of the optical and magneto-optical parameters from granule size are given in $[1,2,4]$.

In the above formulas (1) to (3) a possible distribution of granules by size is not taken into account. At the same time, it is important for the description of optical and magneto-optical spectra of the nanocomposites, since the diagonal components of the dielectric permittivity tensor are responsible for the optical properties, and the nondiagonal for the magneto-optical properties. This is especially true in the near infrared region of the spectrum that is due to intraband transitions $[2,4]$.

Accounting for the size distribution of the granules allows to describe the spectra of the transverse Kerr effect (TKE) more accurately on the p-component is calculated using equations (2-3) according to the formula:

$$
\delta_{P}=\left(A \gamma_{1}+B \gamma_{2}\right) \frac{2 \sin 2 \varphi}{A^{2}+B^{2}}
$$

where

$$
A=\varepsilon_{2}\left(2 \varepsilon_{1} \cos ^{2} \varphi-1\right)
$$

$$
B=\cos ^{2} \varphi\left(\varepsilon_{2}^{2}-\varepsilon_{1}^{2}+1\right)+\varepsilon_{1}-1, \varphi
$$

is the incidence angle of light, $\delta_{p}$ is the parameter of TKE [8]. We considered magnetic nanocomposite for which were optical and magneto-optical parameters experimentally measured [6]. The calculated 
theoretical spectra without size effect of TKE is shown in Fig. 1

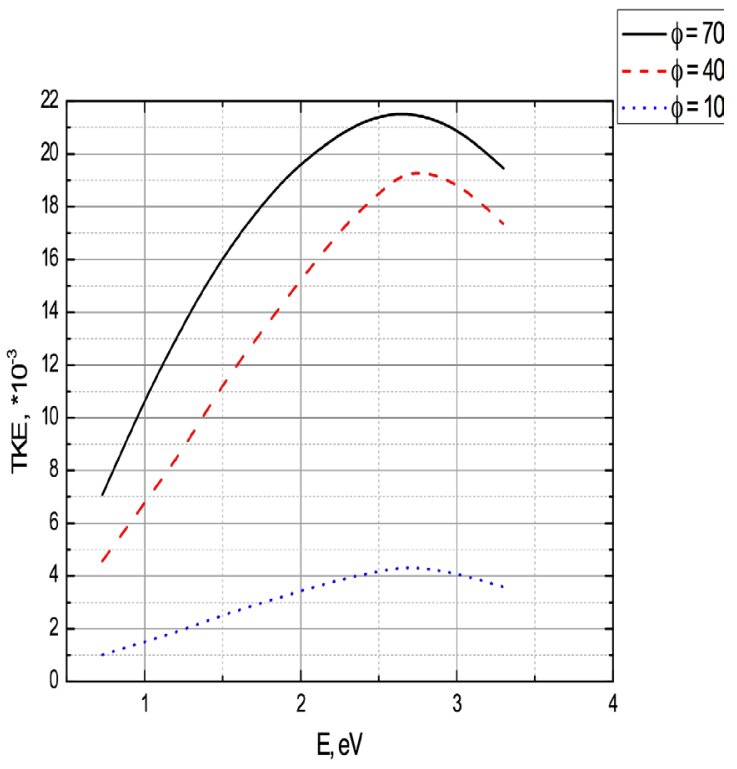

Fig. 1 The calculated spectra of the transversal Kerr effect $\left(\mathrm{Co}_{41} \mathrm{Fe}_{39} \mathrm{~B}_{20}\right) \times\left(\mathrm{SiO}_{2}\right)_{100-\mathrm{x}}$ nanocomposite: $\varphi=70^{\circ}$ - solid line ; $\varphi=40^{0}$ - dash line; $\varphi=10^{\circ}-\operatorname{dot}$ line $(\mathrm{x}=34 \%$, without size effect).

It is clearly seen that the magnitude of this effect strongly depends on the incidence angle of light [Fig.1]. It was shown that the magnitude of the TKE varied more than an order of magnitude [Fig.1].

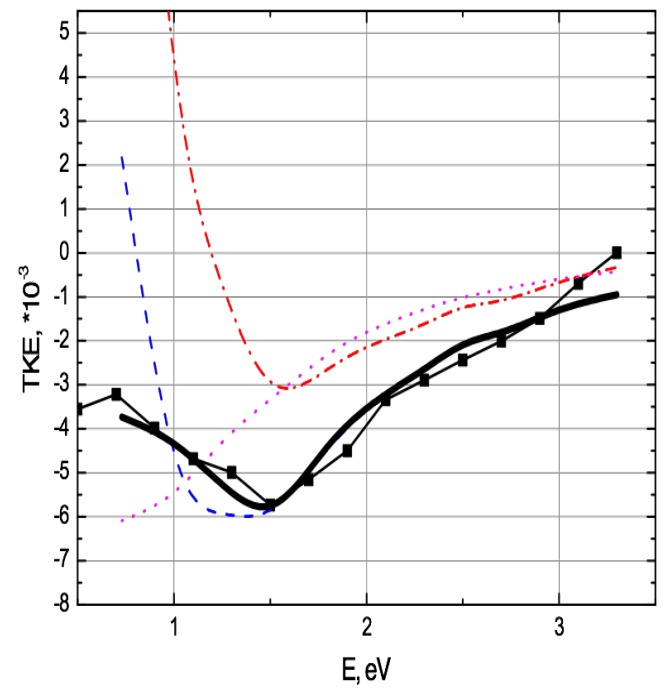

Fig. 2 The calculated spectra of the transversal Kerr effect with the size effect influence compared to the experiment: squares- experimental spectrum; dash dot line-calculated spectra at $\varphi=70^{\circ}$; dash line-calculated spectra taking at $\varphi=77^{\circ}$; dot line-calculated spectra at $\varphi=83^{\circ}(\mathrm{x}=34 \%$, $\mathrm{L}=0.33, \mathrm{r}_{0}=2 \mathrm{~nm}, \mathrm{R}_{\mathrm{s}} / \mathrm{R}_{\text {bulk }}=-2.25$ ) and solid line-calculated spectra taking into account the size effect and particle size distribution at $\varphi=77^{\circ}$.
Fig. 2 shows the calculated spectra of the TKE with the size effect influence compared to the experiment. It was proved that the size effect can change the amplitude, form and sign of the optical and magnetooptical spectra and allows to describe the experimental data well.

For a more accurate description we must take into account the granules distribution by size. Schematically, such a nanocomposite is shown in Fig. 3 . Figure 3 shows one of the variants of layer-by-layer sputtered composites heterogeneity. It is shown that the ferromagnetic granules, which are closer to the previous composite layers have a smaller size compared with those that are in the scope of layers. It is due to the fact that after the deposition of each layer the films are cooled. When we started spraying a new layer, ferromagnetic and dielectric phase falling on the still cold of the previous layer and cooled immediately, which led to the formation of small granules. In proportion the growth of the layer thickness the sample is heated that leads to the formation of larger ferromagnetic grains in the layer. Thus, we can say that composites sputtered layers are not just bulkcomposites with different randomly distributed size granules. In layer-by-layer sputtered composites we can observed a periodic layered structure, the characteristics of which strongly influence the magneto-optical properties of the films.

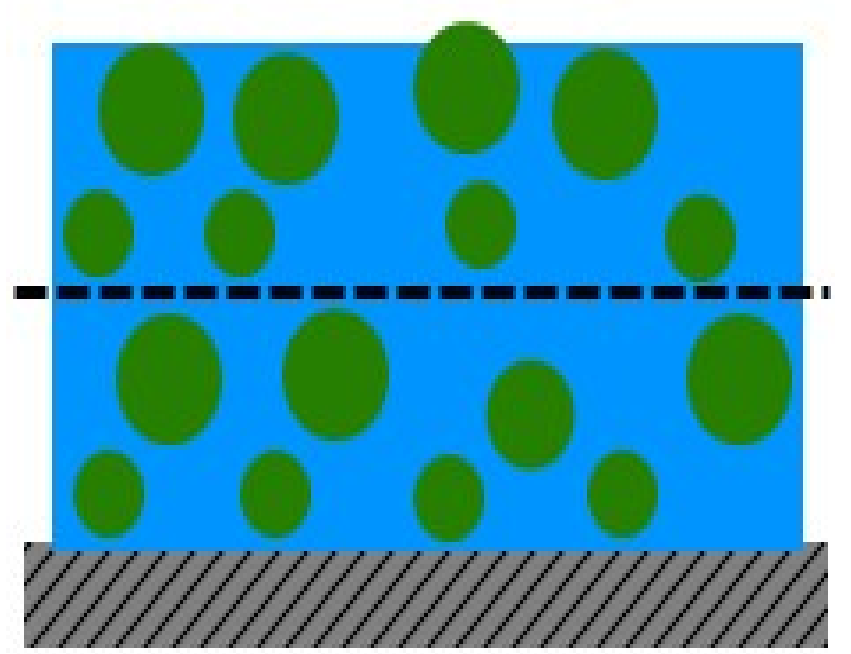

Fig. 3 The particle size distribution in the layers of deposited nanocomposite (dash line - the board between of layer-bylayer sputtered composites heterogeneity).

In the case of a uniform distribution the probability density has the form:

$f_{x}(x)=\left\{\begin{array}{cr}\frac{1}{b-a}, & x \in[a, b] \\ 0, & x \notin[a, b]\end{array}\right.$ 
And the distribution function is :

$F_{x}(x) \equiv P(X \leq x)=\left\{\begin{array}{cc}0, & x<a \\ \frac{x-a}{b-a}, & a \leq x<b \\ 1, & x \geq b\end{array}\right.$

In our version, $a$ is the smallest possible size of a granule, and $\mathrm{b}$ is the largest dimension. Now we denote by $r_{0}$ is the smallest size, $r_{l}$ is the highest size, and $r$ is the current size, then the function size of the granules $R(r)$ can be written in the form:

$R(r)=r_{0}(1+F(r))$.

If the size of the granules is changed two times compared to the original $r_{0}$, the function takes the form:

$$
R(r)=r_{0}\left(1+\frac{r-r_{0}}{r_{1}-r_{0}}\right) .
$$

It is important to note that the uniform distribution (67) is certainly important, but not the general case. Therefore, in consideration of nanocomposites of various compositions, it is important to find the magnitude of the $F(r)$. In figure 2 the solid line presents the spectrum of TKE based on the granules distribution by size and by calculations using the function (9). This particle size distribution allows to describe the corresponding experimental data well (Fig.2).

\section{Conclusion}

Spectra of transversal Kerr effect in $\left(\mathrm{Co}_{41} \mathrm{Fe}_{39} \mathrm{~B}_{20}\right)_{\mathrm{x}}\left(\mathrm{SiO}_{2}\right)_{100-\mathrm{x}}$ nanocomposite have been calculated. Both experimental and theoretical spectra have a local minimum at $\mathrm{E} \sim 1.5 \mathrm{eV}$. This is due to the fact that the size effect in the IR spectral region is connected with intraband transitions. The good interpretation of an experimental data is possible only when the size effect influence is taken into account. Furthermore, for more accurate calculations a distribution function of granules by its size should be used. Thus, the obtained results concerning the size effect and size distribution of the granules allow to improve the description and to explain the amplitude, form and sigh of the optical and magnetooptical spectra in nanocomposites mainly in the near infrared region.

This research was supported by the Russian Foundation for Basic Researches №15-02-02077 and Grant of the RF President for Leading scientific schools SS 8003.2016.2.

\section{References}

1. A.N. Yurasov, RTZ, 1(10), 25 (2016) .
2. G.A. Niklasson, C.G.Granqvist, J. Appl. Phys. 55, 3382 (1984).

3. D. R. Stevens, E. W. Skau, L. N. Downen, M. P. Roman, and L. I. Clarke, Physical Review E 84, 021126 (2011).

4. A.B. Granovsky, E.A. Gan'shina, A.N. Vinogradov, I.K.Rodin, A.N. Yurasov and H.R. Khan, Physics of Metals and Metallography, 91, S52 (2001).

5. C. L. Chien, Xiao John Q., and J. Jiang Samuel, J. Appl. Phys., 73, 5309 (1993).

6. V. Buravtsova, . E. Ganshina, E. Lebedeva, N. Syr'ev, I. Trofimenko, S. Vyzulin, I. Shipkova, S. Phonghirun, Yu. Kalinin, A. Sitnikov, Solid State Phenomena, 168-169, 533 (2011).

7. E. Ganshina, A. Granovsky, V.Gushin, Kuzmichov M., P. Podrugin, A. Kravetz, E. Shipil, Physica A: Statistical Mechanics and its Applications., 241, 45 (1997). (1987) 